\title{
The Stress-Response Network in Animals: Proposals to Develop a Predictive Mathematical Model
}

\author{
David de Pomerai ${ }^{1, *}$, Piyush Madhamshettiwar ${ }^{1}$, Charumathi Anbalagan ${ }^{1}$, Matthew Loose ${ }^{1}$, Mainul \\ Haque $^{2}$, John King ${ }^{2}$, D. Kar Chowdhuri ${ }^{3}$, Pradip Sinha ${ }^{4}$, Bob Johnsen ${ }^{5}$ and David Baillie ${ }^{5}$
}

${ }^{I}$ School of Biology, the University of Nottingham, Nottingham NG7 2RD, UK; ${ }^{2}$ School of Mathematical Sciences, the University of Nottingham, Nottingham NG7 2RD, UK; ${ }^{3}$ Embryotoxicology Section, Indian Institute for Toxicology Research, Lucknow 226001, India and Council of Scientific and Industrial Research (CSIR); ${ }^{4}$ Department of Biological Sciences and Bioengineering, Indian Institutes of Technology Kanpur, Kanpur 208016, India and ${ }^{5}$ Department of Molecular Biology and Biochemistry, Simon Fraser University, 8888 University Drive, Burnaby, British Columbia, Canada V5A 1 S6

\begin{abstract}
Increasing evidence indicates that numerous genetic pathways responding to environmental stress in animals are regulated co-ordinately as well as independently. These stress-response systems should therefore be viewed in holistic terms as a network. As such, their behaviour is susceptible to mathematical modelling using a systems biology approach. This review outlines relevant evidence and describes a newly launched project to develop just such a model using stressresponse data from multiple transgenic strains of C. elegans and D. melanogaster. We hope that our eventual model will be capable of predicting the effects of simple stressor mixtures with reasonable accuracy. To maximise the effectiveness and scope of this model, we appeal for help from colleagues to share reagents and data relevant to this project. We also present preliminary data where RNA interference has implicated the key transcription factor DAF-16 in an unexpected upregulation of cyp-34A9 reporter expression by high cadmium.
\end{abstract}

\section{STRESS RESPONSES AND MIXTURE TOXICITY}

In multicellular organisms, the defensive cellular responses evoked by environmental stresses do not result from simple linear pathways, but rather from a network of interlinked pathways with multiple outputs. This makes it difficult to predict the biological effects of multiple stressors acting together, even though this is the normal situation for industrial pollution of soil or water, where several different contaminants are usually present together. There are few studies and no useful predictive models describing the molecular responses of multicellular organisms to several toxicants acting in concert. This is essentially a systems biology problem, requiring integration of complex molecular and toxicological information. Under the auspices of a Major Award from the UK-India Education and Research Initiative (UK-IERI), we intend to develop an in silico model describing the principal elements of a consensus stress response network (SRN) and its in vivo responses to single stressors, using data from two invertebrate model systems, the nematode Caenorhabditis elegans and the fruit fly Drosophila melanogaster. This model will be used to predict the likely SRN responses to stressor mixtures, and such predictions will then be tested experimentally in both species so that the model can be refined accordingly. Since the SRN core pathways are highly conserved among animal taxa, general features of this model should find wider application in ecotoxi-

\footnotetext{
*Address correspondence to this author at the School of Biology, Nottingham University, Nottingham NG7 2RD, UK; Tel: (0044) 1159513250 ; Fax: (0044) 115951 3251; E-mail: david.depomerai@nottingham.ac.uk
}

cology. The dynamic aspects of this model, and in particular its ability to integrate multiple toxicant inputs for predicting likely SRN outputs, distinguish this from a simple map of the regulatory gene-circuits comprising the SRN.

\section{THE C. ELEGANS STRESS-RESPONSE NETWORK}

The free-living soil nematode, C. elegans, is particularly suitable for joint in vivo and in silico studies - thanks to its small size, anatomical simplicity, ease of culture, rapid lifecycle, unrivalled genetics, complete genome sequence and sophisticated post-genomic technologies (e.g. RNAi) [1]. C. elegans stress-response pathways are studied using molecular biomarkers such as heat-shock proteins [2-5] or metallothioneins [6], and in terms of behavioural or life-cycle parameters such as fertility, growth, motility or feeding [7-9]. C. elegans is used in standard ASTMS tests for soil and water pollution [10,11], as well as for sediment testing [12].

Several C. elegans stress-response pathways are influenced by the daf-2 insulin-like signalling pathway that regulates lifespan [13]. DAF-2 signalling down-regulates the FOXO transcription factor DAF-16, whose targets include the small heat-shock genes (also regulated by HSF-1) [14], the mtl-1 metallothionein gene, the daf-9 and cyp-34A9 (dod-16) P450 genes, and the mitochondrial sod-3 superoxide dismutase gene (whose product helps to inactivate reactive oxygen species, ROS). Each of these DAF-16 targets contributes slightly towards the overall lifespan extension conferred by DAF-16 up-regulation [13]. Although the heatshock factor HSF-1 targets inducible heat-shock genes at elevated temperatures, it also has other functions, as revealed 
by its wide-ranging RNAi phenotypes $[14,15]$. These multipathway links are confirmed by the presence of DAF-16 binding sites [14] as well as ethanol- and hypoxia-response elements [16] within the $h s p-16.1$ promoter, besides the previously identified HSF-1-binding HSEs [17]. These regulatory links show how a single gene can respond to multiple stressors, and how a single stressor can influence multiple outputs (ROS metabolism, metal resistance, chaperone activity and lifespan). Other genes affecting multiple stress responses include the cep-1 p53 orthologue [18].

Several partially independent sub-networks (SNs) can be distinguished within the worm SRN, each regulated principally by one major transcription factor (TF); the heat-shock $\mathrm{SN}$ is activated by HSF-1, the oxidative-stress $\mathrm{SN}$ by zygotic SKN-1 [19], the metallothionein SN by ELT-2 plus metalinduced derepression [20], and the hypoxia SN by HIF-1 (heterodimerised with ARNT) [21]. The primary TF in each SN can be ablated by RNAi, which should facilitate independent modelling of the corresponding SNs. Gene-array studies have shown that DAF-16 primarily up-regulates broad-spectrum detoxification processes [22], including members of the P450, short-chain dehydrogenase/reductase (SDR), UDP glucuronosyltransferase (UGT) and glutathione S-transferase (GST) gene families. This general pattern of response is seen in Drosophila and mouse as well as in $C$. elegans, although lineage-specific diversification of each gene family has occurred within these different animal taxa, such that the genes up-regulated in one species are not necessarily orthologues of those up-regulated in another [23].

\section{THE D. MELANOGASTER STRESS-RESPONSE NETWORK}

Despite its undoubted convenience for ecotoxicological as well as developmental and genetic studies, C. elegans is an organism veritably bristling with defences! Most if not all of its stress-response pathways show evidence of duplication and diversification, perhaps as a consequence of its opportunistic lifestyle as a compost bacterivore. Routinely, it has to cope with osmotic and hypoxic stresses (e.g. when waterlogged), and its natural environment contains multiple metals, organic breakdown products from decomposition processes, and a range of bacterial toxins in its food. This complexity suggests that an additional (and preferably simpler) invertebrate model is also required for this model-building project, particularly if valid extrapolations are to be made for vertebrate systems. An ideal model organism for this purpose is the fruit fly, Drosophila melanogaster, which has a smaller set of stress-response output genes and thus a simpler SRN. Searching the Flybase and Wormbase databases reveals that flies have $<20 \mathrm{P} 450$ genes and $\sim 10$ gst genes compared to $>80$ and 45 , respectively, in $C$. elegans. Amongst other genes in the oxidative stress pathway, C. elegans has 5 superoxide dismutases $(3 \mathrm{Cu} / \mathrm{Zn}$ cytosolic and 2 Mn mitochondrial SODs) and 3 catalases compared to just 2 SODs (1 of each) and 1 catalase in D. melanogaster. These differences point to a simpler basal stress-response network in the fly as compared to the worm. Based on data acquired so far (in the laboratories of DdeP and DKC) using identical stressors, it is likely that Drosophila will prove more sensitive to most if not all toxicants - perhaps reflecting the sophisticated defence systems which have evolved in C. elegans. One advantage of studying two well-characterised in- vertebrate models in parallel is that both the similarities and differences in response to particular toxicants will be highlighted. The former may point to highly conserved aspects of the SRN (which may well also extend to vertebrates), whereas the latter may repay further investigation and comparison with published studies on vertebrate responses (to determine whether Drosophila or C. elegans provides a better model for that part of the SRN). It is worth reiterating that the approach proposed here - involving multiple SRN output genes, multiple doses/time-points and multiple toxicants (both singly and in combination) - is simply not feasible in any vertebrate system. Therefore it will be important to examine carefully how far one can extrapolate predictions of likely mixture toxicity from an invertebrate model to vertebrates (including humans).

\section{CONSTRUCTION OF THE INITIAL MODEL}

Current knowledge of the $C$. elegans SRN encompasses an extensive parts list and at least the outlines of a topology or wiring-diagram model [24]. We know the principal effector genes in each stress-response pathway, and in most cases understand their regulation by stress-activated TFs. However, subtler aspects of effector gene regulation by multiple stressors are only now emerging (e.g. for $h s p-16.1)$ [16]. Our aim is to build an understanding of the underlying control logic [24], initially of each SN separately, and ultimately of the whole SRN. We have started by curating and collating extant knowledge, so as to generate a gene-circuit model that summarises the known SRN - a process successfully applied to mesendoderm specification and heart development [25, 26]. Each link in the network must satisfy 3 criteria. First, the appropriate $\mathrm{TF}(\mathrm{s})$ should have expression patterns consistent with the proposed interaction. Second, experimental perturbation of an upstream TF should evoke an appropriate response from downstream target genes. Third, evidence that the upstream TF binds directly to the target gene promoter is required from other studies of the regulation of that gene. Our initial network model will necessarily be incomplete and will be continuously refined during the project to reflect new data. This will allow construction of a mathematical model, initially describing SNs within the SRN, but working towards a complete model. Our in silico model will incorporate regulatory cross-links between pathways and SNs as they emerge from the experimental data, and will be constructed as a dynamic model describing changes in effector gene expression under various types of stress.

\section{EXPERIMENTAL APPROACH}

Gene-arrays provide one obvious way to explore these stress-responses [27-33]. Gene-array studies of stressresponses in budding [28] and fission [29] yeasts show that overlapping stressor-specific patterns of gene regulation are the norm - superimposed on a core stress response (CSR) involving multiple metabolic genes, which is common to most stressors. The CSR is regulated by MAP kinase signalling via ATF in fission yeast [29], but by several stressspecific pathways in budding yeast [28]. Gene-array studies can provide complete coverage of genome-wide changes in gene expression in response to specific stressors [e.g. 30-33], but are limited by expense, insensitivity to small changes, and sometimes poor reproducibility. Such considerations effectively preclude the routine testing of multiple stressors 
at different doses and time-points - let alone investigating varied combinations of such stressors.

Instead, our experimental data will come mainly from stress-reporter expression assays. We are currently testing a series of single toxicants, but will later progress to simple mixtures of 2 or 3 such toxicants. This approach was originally pioneered using $h s p-16$ and $h s p-70$ reporters [3-5] as biomarkers for both aquatic [34] and soil [35] contamination. Our list of test toxicants will include several metals as well as a variety of widely used pesticides acting on different targets. Currently available stress-inducible reporter strains in C. elegans include: $h s p-16.2$ [36], sod-3, cep-1 [18], cyp35A2 [37], gst-1 [38], mtl-1/ mtl-2 [6] and hsp-16.1 [39] strains. This range of reporters has been greatly expanded thanks to the Baillie group's pioneering genome-wide GFP reporter project [40] to include:- $h s p-60$, gst-4, sod-1/-2/-4, ctl-1/-2, skn-1, hsf-1, 4 P450 genes (cyp-29A2/-31A3/34A9/-35A3) [37] and 3 glutathione peroxidase genes, plus genes involved in DNA repair. However, several large genefamilies are involved in $C$. elegans stress responses ( $45 \mathrm{gst}$, $>80$ cyp and $>10$ glutathione peroxidase genes). From these, we have chosen representatives that are strongly inducible by stress. C. elegans GFP reporters allow in vivo monitoring of expression in real time using a microplate fluorometer, whereas lacZ expression can only be assessed post mortem.

\section{TRANSCRIPTION FACTOR NODES}

Studies to elucidate gene-regulatory networks [e.g. 4143] have emphasised the key role of transcription factor (TF) nodes affecting the expression of multiple downstream effector genes. One recent study modelled the $C$. elegans generegulatory network involved in vulval differentiation, using a combination of TF mutants and GFP-reporter constructs for a range of cell-type-specific effector genes [43]. It is important to know the level and activity of key TFs at these nodes, though it may not be the absolute level of a TF but rather its activity (influenced by ligand-binding, phosphorylation etc) or subcellular location (nuclear versus cytoplasmic) [44] that influences downstream gene expression. To probe the regulatory role of TF nodes in SRN pathways, we will ablate the function of selected TFs by feeding RNAi (e.g. for HSF-1) [45]. The efficacy of RNAi for each TF will be checked by monitoring reporter expression in TF-promoter::GFP fusion strains. Since our main focus is on transcriptional control of the SRN, we do not intend to measure the activity of upstream signalling pathways that activate or repress key TFs. In many cases (e.g. MAP kinase or EGF-like signalling), these pathways are well described and can be incorporated into our in silico model (e.g. the same insulin-like pathway inhibits both SKN-1 and DAF-16) [46] - but in other instances, signals upstream of known TFs remain obscure (e.g. endogenous ligands for steroid receptor-related TFs). Ablation or down-regulation of one SN may also affect the expression of other SNs via cross-talk or compensation between pathways. This should emerge from the RNAi studies, providing evidence for cross-links in the underlying genetic networks. For each of the worm SRN-output genes, we will also compare corresponding genomic sequences between $C$. elegans and $C$. briggsae (related nematodes that diverged $\sim 100$ mya) [47], focussing on the regulatory regions [48] driving our reporter genes and looking for known TF binding sites. Such cross-species bioinformatic comparisons can also reveal conserved sequence blocks likely to represent TFbinding sites [49]. Algorithms [e.g. 50] are available to identify additional regulatory elements for co-regulated genes.

\section{MATHEMATICAL MODELLING OF THE SRN}

The complexity of the SRN as a whole can be adequately addressed only by a systems-biology approach exploiting mathematical tools to generate in silico models that encompass the behaviour of individual network components (e.g. $\mathrm{SNs}$ ) and their integration into a multi-scale formulation of the overall SRN suitable for hypothesis generation and testing, from which in vivo responses to multiple stressors should arise as emergent properties. If such models are to be genuinely predictive, significant quantitative information will be required with regard to network properties. Our experimental programme is carefully structured to enable such information to be extracted, and we hope that our model design will embody a level of complexity commensurate with the development of viable and tractable models that encompass the essential biology without becoming drowned under a flood of extraneous detail.

Even so, the complexity of SRN is such that reliable intuition about how different pathways interact is difficult to establish, and reductionist approaches based on treating each individual $\mathrm{SN}$ in isolation would fail to address the central systems-biology questions as to how the SRN functions as a whole [cf. 51, 52]. Our point of departure is the simplifying assumption that each SN (centred on one TF) functions largely autonomously within the overall SRN. Although this is an over-simplification, it has the methodological advantage of breaking down the overall SRN into more tractable SN units; novel cross-links between these SNs should emerge as the experimental data accumulate. As a first step, we are modelling two of the core $\mathrm{SNs}$, namely the heatshock response (centred on HSF-1) and the oxidative stress response (centred on SKN-1); other SNs will be added to the core model at a later stage. Reporter expression data for incorporation into the model will include both quantitative ( $n$ fold induction relative to zero controls) and dynamic (rate of increase per unit time) information across a range of test doses. Equilibrium and kinetic parameter values associated with each SN can be obtained from the published literature, directly from our experimental data, or inferred indirectly during model exploration and validation. This is a pioneering case study of how state-of-the-art modelling procedures can underpin the development of truly predictive biology.

Insights from both SNs (above) will enable biologically well-grounded models to be developed, initially in the form of nonlinear ordinary differential equations describing the levels of output gene expression within SNs and key TF concentrations, and subsequently the inferred cross-talk between different SNs. By correlating the model predictions against experimental data, model refinement can be pursued, both qualitatively (e.g. introducing further interactions between pathways) and quantitatively (obtaining reliable estimates of the key parameters). Our model(s) will be investigated by:(i) detailed computational simulations (including sensitivity studies on the role of various parameters), (ii) dynamical systems approaches to gain quantitative understanding of the mechanisms underlying observed behaviour and (iii) application of asymptotic treatments to provide simpler model for- 
mulations which still capture the data in a quantitative way, allowing the dominant mechanisms to be uncovered in a systematic fashion.

Our initial SRN model, based on stress-responses to single toxicants, will enable us to predict the likely effects of simple 2-component mixtures. When testing such mixtures, we expect that our initial model will require substantial modification to accommodate the new experimental data, but we are optimistic that less and less adjustment should be required as the model develops. Thus the initial model will be developed and improved through an iterative process of model-based prediction, experimental testing, and subsequent refinement. Model development should not be constrained by the complexity of the mathematics (stochastic, delay and spatio-temporal model formulations will be brought into play, if needed), but only by the desire to provide a realistic description of the biology.

\section{A CASE STUDY: cyp-34A9 AND daf-16}

During the early stages of this project, we were somewhat surprised to observe the induction of a cyp-34A9::GFP reporter (strain BC20306) at high concentrations of several heavy metals (shown for cadmium in Fig. (1), but also seen for copper and for zinc). Cytochrome P450 genes are more typically induced by xenobiotics (33), but cannot detoxify or sequester metals. However, previous studies have identified several cyp genes as targets directly up-regulated by the DAF-16 transcription factor [13, 22, 23], and one such target is cyp-34A9 (dod-16) [13]. This gene is separated from its nearest 5' neighbour cyp-34A10 by less than $300 \mathrm{bp}$ of intergenic sequence, and the integrated transgene construct in strain BC20306 contains only 251 bp of upstream cyp-34A9 promoter sequence (accessible as supplementary Fig. 2 at www.bentham.org/totoxij). Even so, this short region includes a DAF-16 consensus binding site (CATTGT, spanning positions -187 to -191 relative to the transcriptional start site) as well as likely binding sites for other transcription factors. Since RNAi against DAF-16 abolishes the induction of cyp-34A9 by cadmium (Fig. 1) - as well as reducing expression of a daf-16::GFP transgenic strain (TJ356; data not shown) - we conclude that cadmium induction of cyp-34A9 expression is probably mediated through DAF-16 binding to the previously identified upstream site [13]. The cyp-34A9 promoter contains a direct repeat of the nGAAn motif, but our cyp-34A9::GFP reporter strain shows poor heat inducibility at $35^{\circ} \mathrm{C}$, suggesting that this is not a functional HSF binding site. By contrast, the upstream promoter of cyp-35A2 contains a closer match (AAGCTCTT) to the HSF consensus binding site at around -100 ; the fact that a cyp-35A2::GFP reporter strain [37] shows strong heat inducibility at $35^{\circ} \mathrm{C}$ suggests that this site may well be functional, though RNAi against HSF [45] will be needed to confirm this suggestion.

\section{COMMUNITY INVOLVEMENT IN THE SRN PRO- JECT}

This project has ambitious aims, and we do not expect our 3-year study to generate a definitive model whose predictions are invariably accurate. Nevertheless, even a crude model whose predictions roughly approximate to reality for most mixtures tested would still be a significant advance on our current predicament - where mixture toxicity remains largely unpredictable. The more output genes and stressresponse data that can be fed into our in silico model, the more accurate its predictions will be. For this reason, we wish to appeal to all stress biologists, ecotoxicologists and
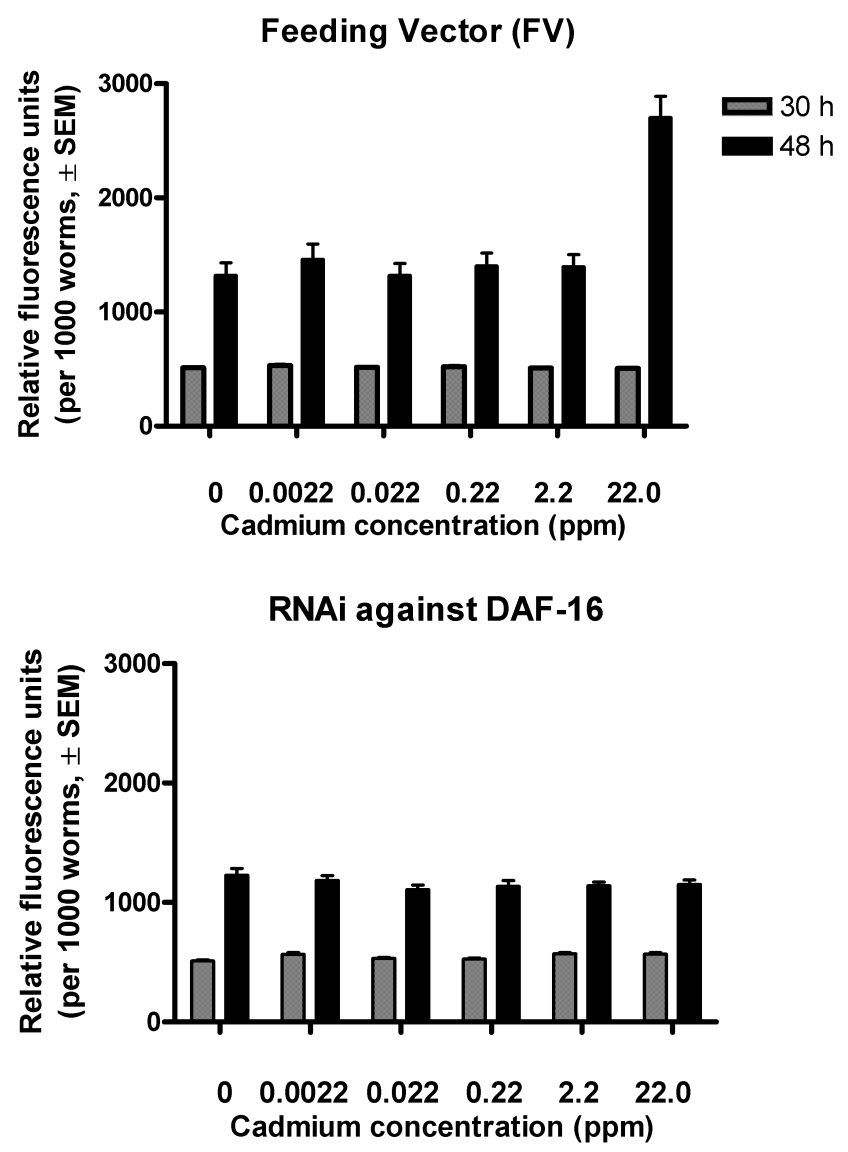

Fig. (1). Effect of RNA interference against DAF-16 on cadmium induction of cyp-34A9::GFP.

Aliquots of $\sim 1000$ young adult BC20306 (carrying 251 bp of upstream promoter from the cyp-34A9 gene [B0213.15] fused to a GFP coding sequence) [40] were dispensed into 24-well plates after feeding overnight on $E$. coli expressing IPTG-induced dsRNA from either a feeding-vector-only control strain (L4440) or a feeding RNAi strain carrying a fragment of the daf-16 (R13H8.1) coding sequence. These bacteria were also present throughout a 48 hour exposure to varying concentrations of cadmium chloride ( 0 to $22 \mu \mathrm{g}$ $\mathrm{ml}^{-1}[=\mathrm{ppm}]$ of $\left.\mathrm{Cd}^{++}\right)$. The contents of each well were transferred to 96-well black non-fluorescent microplatea after $30 \mathrm{~h}$ (grey bars) and $48 \mathrm{~h}$ (black bars), and the level of GFP fluorescence measured in a Perkin-Elmer Victor 1420 fluorometric plate reader. Expression of a daf-16::GFP reporter was reduced by $50 \%$ using RNAi against DAF-16, as compared to the feeding vector only (data not shown). Details of the methodology used can be found in de Pomerai et al. (2003) [45].

molecular biologists working on stress-inducible genes in $C$. elegans or D. melanogaster, to make contact with the authors and to share new findings (e.g. advance online publications) and relevant reagents. We are aware that the selection of transgenic stress-reporter strains available to us is limited in scope, and that other teams have developed similar strains with proven responsiveness to particular chemical 
stressors. Fleshing out our transgene-based SRN with genome-wide gene-array data [27-33] is also vital. We see our dynamic SRN model as a resource for the entire scientific community, and all we can hope to achieve after 3 years will be a first draft, to be updated and refined in the light of future findings so that its predictions become ever more accurate. The support and assistance received from colleagues will allow us to develop a well-rounded model that is more useful to everyone. A dedicated website for this project is now under development, through which we hope to make data summaries for all single-toxicant responses available in a read-only format to interested parties; this site will be accessible via links from the School of Biology website at the University of Nottingham (http://www.nottingham.ac.uk). Other scientists wishing to submit their own data (in a similar format) for inclusion in the process of SRN model development should initially make contact with the corresponding author (david.depomerai@nottingham.ac.uk).

\section{ACKNOWLEDGEMENTS}

The authors would like to thank Declan Brady for technical assistance and their colleagues for helpful discussions. The financial support of the UK-India Education and Research Initiative (UK-IERI) is gratefully acknowledged. The Caenorhabditis Genetics Centre (CGC, University of Minnesota, USA) is thanked for supplying strain TJ356.

\section{SUPPLEMENTARY MATERIAL}

Supplementary material can be viewed at: http://www. bentham.org/open/totoxij.

\section{REFERENCES}

[1] Kamath RS, Fraser AG, Dong Y, et al. Systematic functional analysis of the Caenorhabditis elegans genome using RNAi. Nature 2003; 421: 231-7.

[2] Candido EPM, Jones D. Transgenic Caenorhabditis elegans strains as biosensors. Trends Biotechnol 1996; 14: 125-9.

[3] Guven K, Duce JA, de Pomerai DI. Evaluation of a stress-inducible transgenic nematode strain for aquatic toxicity testing. Aquat Toxicol 1994; 29: 119-37.

[4] Stringham E, Candido EPM. Transgenic hsp16-lacZ strains of the soil nematode Caenorhabditis elegans as biological monitors of environmental stress. Environ Toxicol Chem 1994; 13: 1211-20.

[5] Power RS, David HE, Mutwakil MHAZ, et al. Stress-inducible transgenic nematodes as biomonitors of soil and water pollution. $\mathbf{J}$ Biosci 1998; 23: 513-26.

[6] Swain SC, Keusekotten K, Baumeister R, Sturzenbaum SR. $C$. elegans metallothioneins: new insights into the phenotypic effects of cadmium toxicosis. J Mol Biol 2004; 341: 951-9.

[7] Dhawan R, Dusenbery DB, Williams PL. A comparison of metalinduced lethality and behavioural responses in the nematode Caenorhabditis elegans. Environ Toxicol Chem 2000; 19: 3061-7

[8] Jones D, Candido EPM. Feeding is inhibited by sublethal concentrations of toxicants and by heat stress in the nematode Caenorhabditis elegans: relationship to the cellular stress response. J Exp Zool 1999; 284: 147-57.

[9] de Pomerai DI, Dawe A, Djerbib L, Allan J, Brunt G, Daniells C. Growth and maturation of the nematode Caenorhabditis elegans following exposure to weak microwave fields. Enzyme Microb Technol 2002; 30: 73-9.

[10] Freeman MN, Marse TJ, Williams PL. Establishment of quality assurance procedures for aquatic toxicity testing with the nematode Caenorhabditis elegans. In: Little EE, DeLonay AJ, Greenberg BM, Eds. Environ Toxicol Risk Assess ASTM STP 1333. American Society for Testing and Materials, West Conshohcken, PA 1998; Vol. 7.

[11] Freeman MN, Peredney CL, Williams PL. A soil bioassay using the nematode Caenorhabditis elegans. In: Henshel DS, Black MC,
Harrass MC, Eds. Environmental Toxicology and Risk Assessment: standardisation of biomarkers for endocrine disruption and environmental assessment. Env Toxicol Risk Assess. ASTM STP 1364. American Society for Testing and Materials, West Conshohcken, PA 1999; Vol. 8: pp. 305-18.

[12] Traunspurger W, Haitzer M, Hoss S, Beier S, Ahle W, Steinberg C. Ecotoxicological assessment of aquatic sediments with Caenorhabditis elegans (Nematoda) - a method for testing liquid medium and whole-sediment samples. Environ Toxicol Chem 1997; 16: 245-50.

[13] Murphy CT, McCarroll SA, Bargmann CL, et al. Genes that act downstream of DAF-16 to influence the lifespan of C. elegans. Nature 2003; 424: 277-84.

[14] Hsu A-L, Murphy CT, Kenyon C. Regulation of aging and agerelated disease by DAF-16 and heat-shock factor. Science 2003; 300: $1142-5$

[15] Walker G, Thompson FJ, Brawley A, Scanlon T, Devaney E. Heat shock factor functions at the convergence of the stress response and developmental pathways in Caenorhabditis elegans. FASEB J 2003; 17: 1960-2.

[16] Hong M, Kwon JY, Shim J, Lee J. Differential hypoxia response of hsp-16 genes in the nematode. J Mol Biol 2004; 344: 369-81.

[17] Candido EPM, Jones D, Dixon DK, Graham RW, Russnak RH, Kay RJ. Structure, organisation and expression of the 16-Kda heat shock family of Caenorhabditis elegans. Genome 1989; 31: 690-7.

[18] Derry WB, Putzke AP, Rothman JH. Caenorhabditis elegans p53: role in apoptosis, meiosis and stress resistance. Science 2001; 294: 591-5.

[19] An J-H, Blackwell T. SKN-1 links C. elegans mesendodermal specification to a conserved oxidative stress response. Genes Dev 2003; 17: 1882-93.

[20] Moilanen LH, Fukushige T, Freedman JH. Regulation of metallothionein gene transcription: identification of upstream regulatory element and transcription factors responsible for cell-specific expression of metallothionein genes from Caenorhabditis elegans. J Biol Chem 1999; 274: 29655-65.

[21] Jiang H, Guo R, Powell-Coffman JA. The Caenorhabditis elegans hif-1 gene encodes a bHLH-PAS protein that is required for adaptation to hypoxia. Proc Natl Acad Sci USA 2001; 98: 7916-21.

[22] McElwee J, Schuster E, Blanc E, Thomas J, Gems D. Shared transcriptional signature in Caenorhabditis elegans dauer larvae and long-lived daf-2 mutants implicates detoxification system in longevity assurance. J Biol Chem 2004; 279: 44533-43.

[23] Gems D, McElwee J. Broad-spectrum detoxification: the major longevity assurance process regulated by insulin/IGF-1 signalling? Mech Ageing Dev 2005; 126: 381-7.

[24] Schlitt T, Brazma A. Modelling gene networks at different organisational levels. FEBS Lett 2005; 579: 1859-66.

[25] Loose M, Patient R. A genetic regulatory network for Xenopus mesendoderm formation. Dev Biol 2004; 271: 467-78.

[26] Peterkin T, Gibson A, Loose M, Patient R The roles of GATA-4, -5 and -6 in vertebrate heart development. Semin Cell Dev Biol 2005; 16: 83-94.

[27] Kim SK, Lund J, Kiraly M, et al. A gene expression map for Caenorhabditis elegans. Science 2001; 293: 2087-92.

[28] Gasch AP, Spellman PT, Kao CM, et al. Genomic expression programs in response of yeast cells to environmental changes. Mol Biol Cell 2000; 11: 4241-57.

[29] Chen D, Toone WM, Mata J, et al. Global transcriptional responses of fission yeast to environmental stress. Mol Biol Cell 2003; 14: 214-29.

[30] Cui Y, McBride SJ, Boyd WA, Alper S, Freedman JH. Toxicogenomic analysis of Caenorhabditis elegans reveals novel genes and pathways involved in the resistance to cadmium toxicity. Genome Biol 2007; 8: R122.

[31] Kwon JY, Hong M, Choi MS, et al. Ethanol-response genes and their regulation analyzed by a microarray and comparative genomic approach in the nematode Caenorhabditis elegans. Genomics 2004; 83: 600-14

[32] Custodia N, Won SJ, Novillo A, Wieland M, Li C, Callard IP. Caenorhabditis elegans as an environmental monitor using DNA microarray analysis. Ann NY Acad Sci 2001; 948: 32-42.

[33] Menzel R, Yeo HL, Rienau S, Li S, Steinberg CE, Sturzenbaum SR. Cytochrome P450s and short-chain dehydrogenases mediate the toxicogenomic response of PCB52 in the nematode Caenorhabditis elegans. J Mol Biol 2007; 370: 1-13. 
[34] Mutwakil MHAZ, Reader JP, Holdich DM, et al. Use of stressinducible transgenic nematodes as biomarkers of heavy metal pollution in water samples from an English river system. Arch Environ Contam Toxicol 1997; 32: 146-53.

[35] Power RS, de Pomerai DI. Effect of single and paired metal inputs in soil on a stress-inducible transgenic nematode. Arch Environ Contam Toxicol 1999; 37: 503-11.

[36] Link CD, Cypser JR, Johnson CJ, Johnson TE. Direct observation of stress response in Caenorhabditis elegans using a reporter transgene. Cell Stress Chaperon 1999; 4: 235-42.

[37] Menzel R, Bogaert T, Achazi R. A systematic gene expression screen of Caenorhabditis elegans cytochrome P450 genes reveals CYP35 as strongly xenobiotic inducible. Arch Biochem Biophys 2001; 395: 158-68.

[38] Leiers B, Kampkotter A, Grevelding CG, Link CD, Johnson TE, Henkle-Duhrsen K. A stress-responsive glutathione S-transferase confers resistance to oxidative stress in Caenorhabditis elegans. Free Radical Biol Med 2003; 34: 1405-15.

[39] David HE, Dawe AS, de Pomerai DI, Jones D, Candido EPM, Daniells C. Construction and evaluation of a transgenic hsp16GFP-lacZ Caenorhabditis elegans strain for environmental monitoring. Environ Toxicol Chem 2003; 22: 111-8.

[40] McKay SJ, Johnsen R, Khattra J, et al. Gene expression profiling of cells, tissues and developmental stages of the nematode $C$. elegans. Cold Spring Harbor Symp Quant Biol 2003; 68: 159-69.

[41] Davidson E, Rast J, Oliveri P, et al. A genomic regulatory network for development. Science 2002; 295: 1669-78.

[42] Levine M, Davidson E. Gene regulatory networks for development. Proc Natl Acad Sci USA 2005; 102: 4936-42.

[43] Inoue T, Wang M, Ririe T, Fernandes J, Sternberg P. Transcriptional network underlying Caenorhabditis elegans vulval development. Proc Natl Acad Sci USA 2005; 102: 4972-7.
[44] Zou J, Guo Y, Guettouche T, Smith D, Voellmy R. Repression of heat shock transcription factor HSF1 activation by HSP90 (HSP90 complex) that forms a stress-sensitive complex with HSF1. Cell 1998; 94: 471-80.

[45] de Pomerai DI, Smith B, Dawe A, et al. Microwave radiation can alter protein conformation without bulk heating. FEBS Lett 2003; 543: 93-7.

[46] Tullet JM, Hertweck M, An JH, et al. Direct inhibition of the longevity-promoting factor $\mathrm{SKN}-1$ by insulin-like signalling in $C$. elegans. Cell 2008; 132: 1025-38.

[47] Stein LD, Bao Z, Blasiar D, et al. The genome sequence of Caenorhabditis briggsae: a platform for comparative genomics. .PLoS Biol 2003; 1: 166-92.

[48] Kent W, Zahler A. Conservation, regulation, synteny, and introns in a large-scale $C$. briggsae-C. elegans genomic alignment. Genome Res 2000; 10: 1115-25.

[49] Wingender E, Chen X, Hehl R, et al. Transfac: An integrated system for gene expression regulation. Nucleic Acids Res 2000; 28: 316-9.

[50] Liu Y, Liu XS, Wei L, Altman RB, Batzoglou S. Eukaryotic regulatory element conservation analysis and identification using comparative genomics. Genome Res 2004; 14: 451-8.

[51] Ge H, Walhout A, Vidal M. Integrating 'omic' information: a bridge between genomics and systems biology. Trends Genet 2003; 19: 551-60.

[52] Kolchanov N, Podkolodnaya OA, Ananko EA, et al. An integrated computer system for studying the regulation of eukaryotic gene expression. Mol Biol 2004; 38: 58-9.

(c) de Pomerai et al.; Licensee Bentham Open.

This is an open access article licensed under the terms of the Creative Commons Attribution Non-Commercial License (http://creativecommons.org/licenses/ by-nc/3.0/) which permits unrestricted, non-commercial use, distribution and reproduction in any medium, provided the work is properly cited. 